\title{
Real-time focus and overlay measurement by the use of fluorescent markers
}

\author{
Diederik Maas* and Erwin van Zwet
}

TNO, Stieltjesweg 1, 2628 CK Delft, The Netherlands

\begin{abstract}
In lithography, overlay control is getting increasingly complex. Advanced Process Control (APC) is introduced to minimize excursions from the process window for the present exposure. APC uses metrology data of previously exposed wafers, hence, there is always a delay of tens of minutes before the required information is available. This paper proposes the combination of a patterned expose beam and a patterned fluorescent marker on a wafer to generate a fluorescent signal that carries real-time information of the focus and/or position error of the expose pattern with the pattern on the wafer. A practical realization requires some changes to the exposure process, stepper design and reticle lay-out. Firstly, a matched pair of markers on the wafer and reticle is required. Secondly, the generated fluorescent signal must be measured, for example with a (spectrally filtered) photon counter close to the expose area of the wafer. At last, the markers from the previous lithography step shall, after development, be filled with fluorescent material. This deposition requires an additional process step. Photon budget calculations suggest an overlay measurement accuracy of less than a tenth of a nm (real-time).
\end{abstract}

Keywords: Overlay, focus, lithography, image placement, metrology integration, advanced process control, multiple patterning, fluorescence, processing

\section{INTRODUCTION}

In lithography, improving overlay (OVL) control including process dependencies below a level of $5 \mathrm{~nm}$ is a complex challenge ${ }^{1}$. As the IC industry is driven by pattern shrink, the need for accurate overlay control is ever more critical ${ }^{2}$. Felix et al. identified five categories contributing to on-product overlay: scanner, process, reticle, metrology and APC $^{3}$. Mulkens et al. introduced a holistic lithography systems architecture that maximizes the OVL improvement that is obtainable with $\mathrm{APC}^{4}$.

This paper presents a method to sample OVL real-time, i.e. during expose, aiming to tackle scanner, reticle and process contributions to on-product $\mathrm{OVL}^{5}$. It is proposed to use fluorescent material in at-product-pitch markers, e.g. positioned in the scribe line, to generate real-time OVL signals. That fluorescent markers can indeed be used to measure the positioning and imaging performance of a lithography scanner is shown by Brunner et el. in the 80s of the precious century $^{6-8}$. Albeit addressing lens heating, Brunner's experiments did not use at-product-pitch features in the markers. Multiple patterning was invented only some 20 years later $^{9}$, which, combined with the introduction of more extreme pupil filling patterns enhanced the need for better control over pattern placement errors (PPE) and OVL.

Here, we aim to show that signals from at-product-pitch markers provide insight in expose-beam-induced overlay errors, as well as pattern placement errors, e.g. as caused by lens heating. The method is applicable to multiple-patterning lithography with DUV, EUV and even electron beam projection exposures ${ }^{10}$, provided similar pupil illumination is used in consecutive steps and a suitable fluorophore is available.

We foresee an accelerated yield-ramp, enabled by real-time OVL data, since these could characterize correctable OVL errors. Once the yield is at target level and there is no need to measure OVL real-time, production could continue without the fluorescent markers. The proposed method is expected to be most economical for the production of small series of ICs at latest patterning node, e.g. ASIC. 


\subsection{Overlay markers}

We briefly review the design and limitations of conventional OVL markers and then turn to the features of fluorescent OVL markers. The term "overlay" is used to refer to the pattern-to-pattern alignment in a sequence of manufacturing steps. The term "pattern placement error" (PPE) refers to the displacement of a pattern feature relative to its design coordinates. In order for the final chip device to function correctly, OVL, focus and PPE should be accurate enough such that the various patterns of e.g. lines, contacts, and transistors are properly aligned and connected.

Historically, overlay markers are used with pitches or critical dimensions in the order of 10 micron. These markers allowed alignment of a projected pattern to patterns on the wafer by either imaging of the markers or measuring diffraction coming from the markers. Today's high-end overlay measurement systems are based on the simultaneous detection of refracted visible light in several diffraction orders from the marker, see e.g. Fig. 5 in Mulkens's paper ${ }^{4}$. The use of visible light and conventional optics makes it impossible to shrink the pitch of the markers to the pitch that corresponds to the lithography node of the device.

The exact location of an on-product-pitch pattern at the nanometer scale depends on the aberrations of the projecting optics. These aberrations are temperature dependent, and hence vary over time due to the expose-process-dependent lens heating. For today's extreme pupil illumination settings, the expose beam energy density can be very localized in the Fourier plane. As a consequence, the lens-heating-induced shift of conventional markers is no longer equal to the shift of structures printed at the device pitch. And because the printing of an isolated line feature contains additional frequency components compared to a dense periodic structure, isolated lines at (or close to) product-pitch shift differently as well. As a result the conventional markers are not always reliable predictors for the location of the process structures. During yield ramp, part of the time, effort and cost are spent on learning of and getting control over these effects.

This paper introduces a method (and sketches part of the involved instrumentation) to measure OVL, PPE and focus in a lithographic process real-time, i.e. in-line. The method uses new markers that are based on at-product-pitch-sized features $^{5}$. Major advantage of the novel method is that dynamic and local deformations in wafer position and shape as well as in the projected reticle pattern are measured. This unique information will add to the OVL and PPE data from conventional metrology, and thus enable a faster yield ramp.

Furthermore, potentially a real-time overlay signal can be used as feed-back in the control loop of the motion of reticle and wafer stage for improved overlay during exposure. The current best-practice is based on feed-forward correction of positioning errors ${ }^{11}$.

\subsection{Real-time OVL measurement}

A practical realization requires some changes in and additions to the exposure process, stepper design and reticle lay-out. Firstly, a matched pair of markers on the wafer and reticle is required. Secondly, the markers from the previous lithography step shall, after development, be filled with fluorescent material. At last, the generated fluorescent signal must be measured.

To illustrate a possible process flow, Fig. 1 shows cross-sectional images of a wafer that is patterned using a modified Litho-Etch-Litho-Etch double-patterning (DP) process. The first lithographic step prints both the customer part and (atproduct-pitch) markers in the scribe line. After development and etch, the markers are filled with fluorescent material. During the second lithographic exposure, the fluorescent signal measures OVL. PPE and focus. Typically, more fluorescence signal is generated when the overlay is not good. The exact implementation will be slightly different for each variant of double patterning (e.g. Litho-Litho-Etch, Litho-Etch-Litho-Etch, etc.). Furthermore, the real-time metrology data can be related to a-posteriori measured overlay and focus ${ }^{1-4}$. 




Figure 1. Simplified dual-patterning process-flow for real-time overlay measurement using fluorescent markers. The fluorescent marker is exposed to a pattern that is shifted over approximately a quarter pitch, whereas for the customer part the second expose pattern is shifted over half the pitch. The fluorescent signal is a direct and real-time measure of the pattern placement error.

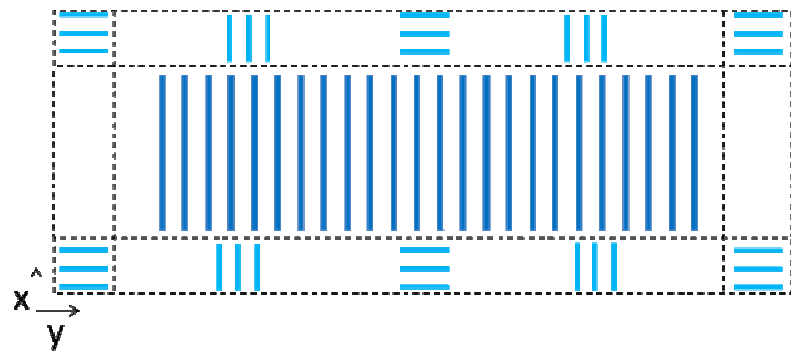

$1^{\text {st }}$ pattern



$2^{\text {nd }}$ pattern

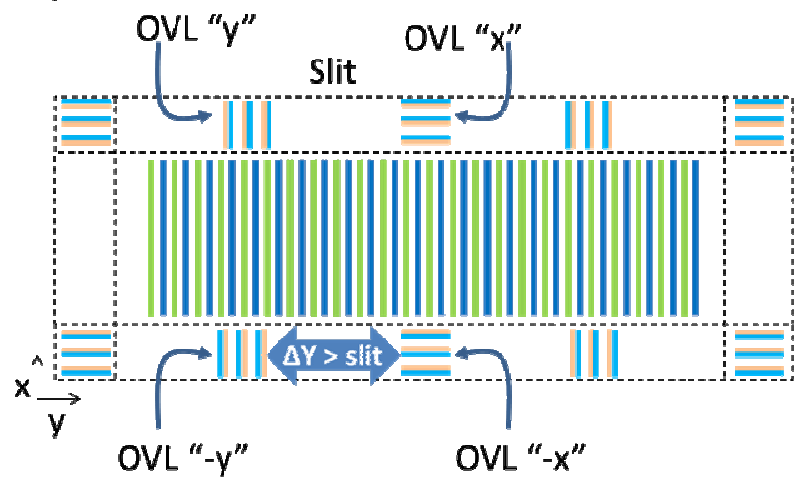

Full pattern

Figure 2. Illustration suggesting a possible arrangement of marker pairs in the scribe lanes. The scan direction of the stages is $\mathrm{Y}$. The alternating marker orientation enables sampled measurements of the $\mathrm{X}$ - and Y-OVL. 


\subsection{Sketch of marker design on reticle pair}

Figure 2 shows an arrangement of a set of marker pairs on the first and second mask, as well as the resulting pattern after both exposures. A matched pair of markers on the reticle and wafer is required to measure the overlay of the projected image of the reticle relative to the pattern on the wafer. The marker on the reticle patterns the expose beam. On the wafer, a fluorescent marker with a complementary pattern generates a fluorescent signal during exposure. The fluorescence strength represents the (error in the) overlay, as already shown by experimental data from $1985^{6}$. The slit of the expose beam is a few $\mathrm{mm}$ wide. Hence the markers shall be separated by at least the slit width to avoid cross-talk between X- and Y-OVL signals. Consequently, for a field size of $33 \mathrm{~mm}$, the number of marker pairs is limited to about ten.

\subsection{Fluorescent markers}

A suitable fluorophore should be compatible with the lithography step and all further processes, generate photons efficiently, not bleach during the exposure and be applied in a relatively thin layer of less than 50-100 nm. Potential inorganic candidates are CdSe quantum dots in an organic matrix or (a mix of) Lanthanides ( $\mathrm{Tb}, \mathrm{Gd}, \mathrm{Er}$ of $\mathrm{Pr}$ ) in a solgel matrix. Furthermore, also many organic fluorophores have been developed during the last decade as markers in biology, and although a topic for further research, we anticipate that some of those will be sufficiently bleach-resistant against DUV and EUV and compatible with further processing in a semiconductor factory.

\section{Measuring overlay, PPE and focus in a single direction}

Figure 3 shows the normalized fluorescence intensity as a function of the relative displacement of a patterned expose beam (green rectangles in the insets) that is projected on a fluorescent marker (represented by the yellow rectangles in the insets) on a wafer. Maximum fluorescence intensity (100\%) occurs when marker and patterned beam have full overlap. This occurs for overlay errors equal to the marker size, i.e. typically tens to hundreds of nanometers. The exact pattern design is free, yet will typically contain a grating, if it were only to increase the sensitivity of the measurement by maximize the amount of (barely) overlapping edges. An advanced spatial design of the matched pair of markers can create a fluorescent signal strength that is related or even proportional to the error in the overlay of the expose pattern with the layer that contains the fluorescent marker.

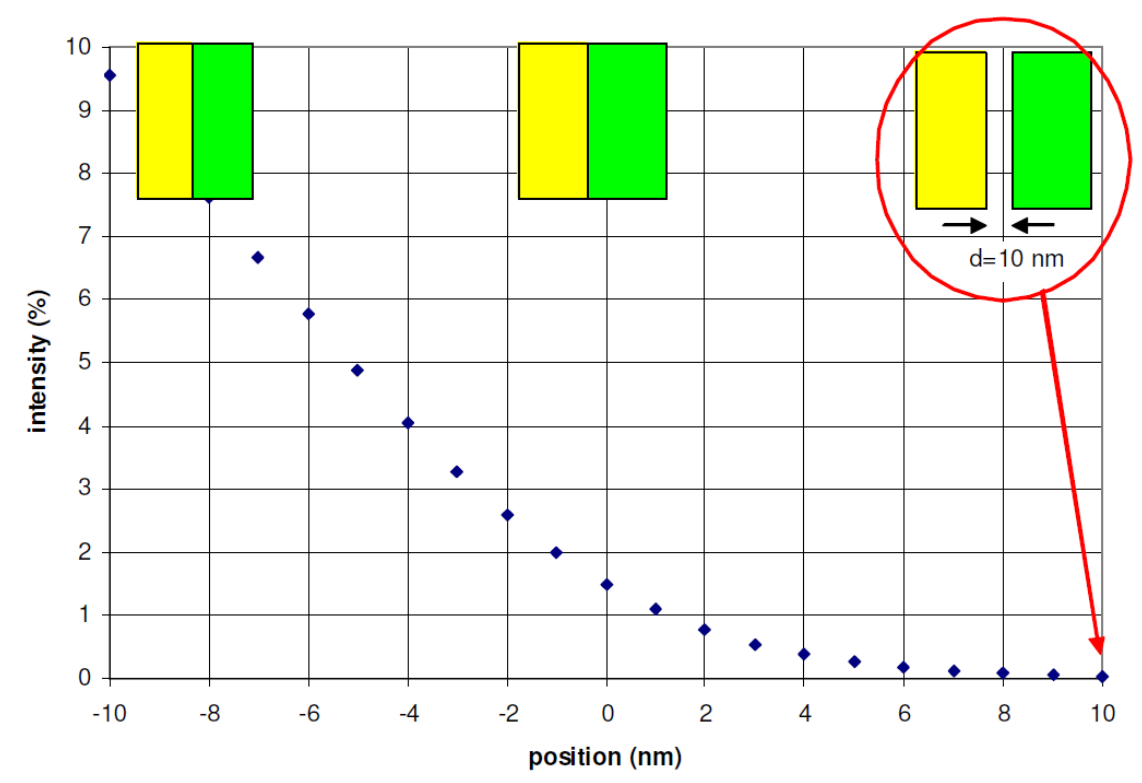

Figure 3. The normalized fluorescence signal ("Intensity in \%") as a function of overlay error ("position [nm]"). In the insets, the fluorescent marker is schematically represented by the yellow rectangles, and the patterned expose beam by the green rectangles.

\section{Measuring overlay, PPE and focus in all directions}

The simple marker design of Figure 3 senses overlay in only one direction. Four markers are needed for monitoring OVL in all directions. Care shall be taken in the fluorescence detection (as discussed in below) to avoid cross-talk 
between the different signals. For example, a complete pattern can consist of multiple smaller markers that each addresses a direction of the overlay error in a specific part of the exposed die. Figure 4 shows the difference signal from a pair of fluorescent markers as a function of the overlap in two directions. The response of the fluorescence on the relative position suggests a possible use of the signal in feedback loop on stage position control.

In a practical embodiment, it is not trivial to measure the signal from two markers simultaneously. Figure 2 shows a possible solution: the markers for the $+X$ and $-X$ direction are arranged in opposite scribe lanes, assuming that their fluorescence can be detected independently. Another solution could be to select a different emission wavelength of the fluorescent material for each direction. In the latter case, it seems preferred to add adequate spectral color filters to the fluorescence detector for each direction. To monitor the OVL in all $\pm X$ and $\pm Y$ directions, markers with alternating orientation can be used either sequentially, as is illustrated in Figure 2 by separating them by more than the expose beam slit width (which reducing the sampling frequency) or by using four flourophores, each having a different emission wavelength and a conjugated detector.

Optimizing marker design for overlay, PPE and focus accuracy and sensitivity

Next to their arrangement, also the exact pattern of the markers is related to the obtainable accuracy and sampling frequency. The study after the impact of the 3D marker lay out on the signal strength is outside the scope of this paper. However, recently the design and performance of overlay marker designs for both diffraction and imaging based overlay measurements targeting sub-20 nm patterning nodes is discussed recently by Blancquaert ${ }^{12}$. Bhattacharyya et al. show that the stepper focus can be measured over a wide range with an asymmetric marker that has a large side-wall angle ${ }^{13}$. The insights gained in marker design from these papers will be considered when optimizing the 3D shape of fluorescent markers for overlay, PPE and/or focus.

For today's tight process windows, the contrast in the arial image, or equivalently the value of the NILS, is strongly dependent on the exact focus ${ }^{4}$. The contrast of the projected grating of the markers, and hence the strength of the fluorescent signal, depends on the NILS and hence on focus. At the moment of writing this paper, it is still open how to resolve the crosstalk between focus and overlay. Perhaps it is possible to design a marker with sufficiently low crosstalk, perhaps experimental procedures can be developed to avoid or even use the mixing of these signals, similarly to the Yieldstar marker designs ${ }^{13}$.
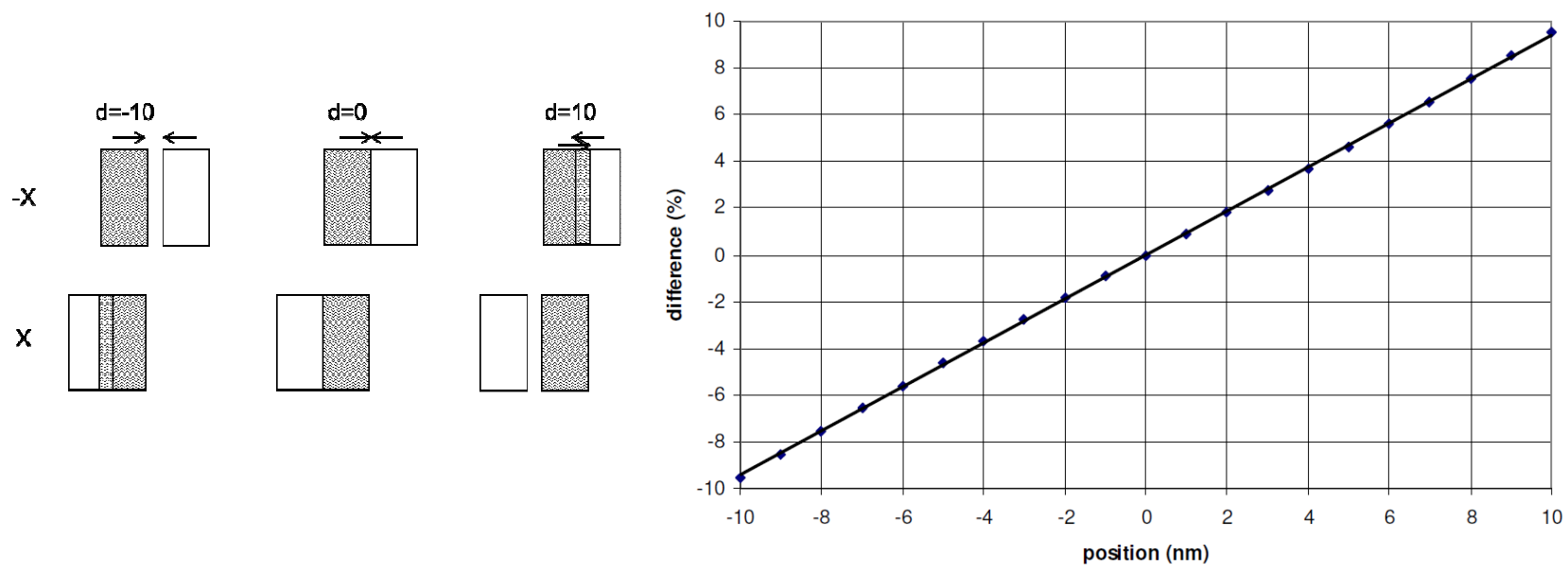

Figure 4. left panel: illustration of overlay for relative positions $d=-10,0$ and $10 \mathrm{~nm}$ of fluorescent markers in $-\mathrm{X}$ and $+\mathrm{X}$ direction with the expose beam. Right panel: The difference in fluorescence signal strength ("Difference in \%") from a marker pair, monitoring e.g. $+\mathrm{X}$ and $-\mathrm{X}$, as a function of overlay error ("position [nm]").

\section{Sampling frequency of the overlay measurement}

In the expose tool, the patterned light beam is limited to a slit of only a few mm wide. The projected image size of the reticle is $26 \times 33 \mathrm{~mm}$. If the markers are to be separated by at least the slit width (estimated at $3 \mathrm{~mm}$ ), the number of samples of OVL \& focus is limited to about ten per die. Ergo, each direction can be sampled only a few times per field. For an expose tool with a typical throughput of $150 \mathrm{WPH}$, exposing 300-mm wafers with 100 expose fields each, the sampling frequency is estimated at $50 \mathrm{~Hz}$. 


\section{Embodiments for the fluorescence detection}

The work by Brunner et al. showed that it is feasible to detect the fluorescent signal from the marker in a dry scanner ${ }^{6-8}$. Since then, changes are made to the expose area in the scanner that block a direct line of sight. For instance, immersion lithography involves an immersion hood. For today's scanners, a few options for the integration of the fluorescence detection somewhere in the projection system appear feasible. In this paper, we will not work out the performance of these detectors in detail. In one embodiment the detectors are placed close to the wafer, e.g. integrated in the immersion hood. A potential advantage of having the detectors so close to the die is that the signal form the markers in opposing scribe lanes can be recorded independently. In another embodiment use is made of the high collection efficiency of the high-NA projection optics column. Options for detecting the collected fluorescence are available: e.g. by integrating sensors on the reticle blades, close to the conjugated plane of the focus, or e.g. in one of the mirrors of a catadioptric projection column, provided the reflective coating for the $193 \mathrm{~nm}$ light can be made transparent for the fluorescence wavelengths.

\section{PREDICTIONS BY THE PHOTON BUDGET MODEL}

\subsection{Fluorescent overlay markers at product pitch $\&$ Measuring overlay and focus using at resolution markers}

This paper proposes the use of markers filled with scintillating material, as those can be made equally small and at the same pitch as the finest process structures. This is enabled by the readout of the markers with similar (or even the same) optics and at the same wavelength during the next exposure as was used for the first exposure. The use of periodic markers with a pitch equal to the (single pattern) device structures imposes some limitations to the marker design. The preferred option is making the width of the scintillating markers smaller than the halve pitch. This can be done by the proper etching steps and doesn't conflict with the resolution capabilities of the lithographic tool. This reduction in the width of the markers allows to be sensitive to a specific direction of misalignment as is illustrated in Figure 5.

The width of the marker can be chosen to optimize either the measurement signal with a marker width approaching the half pitch, or increasing the measurement range by making the marker as small as possible. In Figure 5 a $15 \mathrm{~nm}$ marker is shown with a $20 \mathrm{~nm}$ half pitch structure. The fraction of the illumination light as function of misalignment to the marker is displayed in Figure 6 for a worst-case NILS of 1.6. As can be seen in both Figure 5 and 6 , about $23 \%$ of the illumination light is projected on the scintillating marker. The relative change as function of misalignment is about $0.4 \%$ per $0.1 \mathrm{~mm}$ misalignment. For a more practical NILS of 3, the sensitivity is about $1.7 \%$ per $0.1 \mathrm{~nm}$ overlay error.

\section{At-product-pitch marker OVL accuracy estimate}

A simple photon budget has been made to estimate the detection sensitivity in the shot noise limit. In a DUV system at expose dose of $20 \mathrm{~mJ} \mathrm{~cm}^{-2}$ the detector records $\sim 10^{5}$ photons from a $5 \times 5 \mu \mathrm{m}$ marker with $15 \mathrm{~nm}$ lines at $40 \mathrm{~nm}$ pitch, see Figure 5. Hence, fluorescent markers enable real-time OVL detection with accuracy of $0.02 \mathrm{~nm}(1 \sigma)$ for the best-case of shot-noise-limited detectors in expose systems with NILS $=3$.

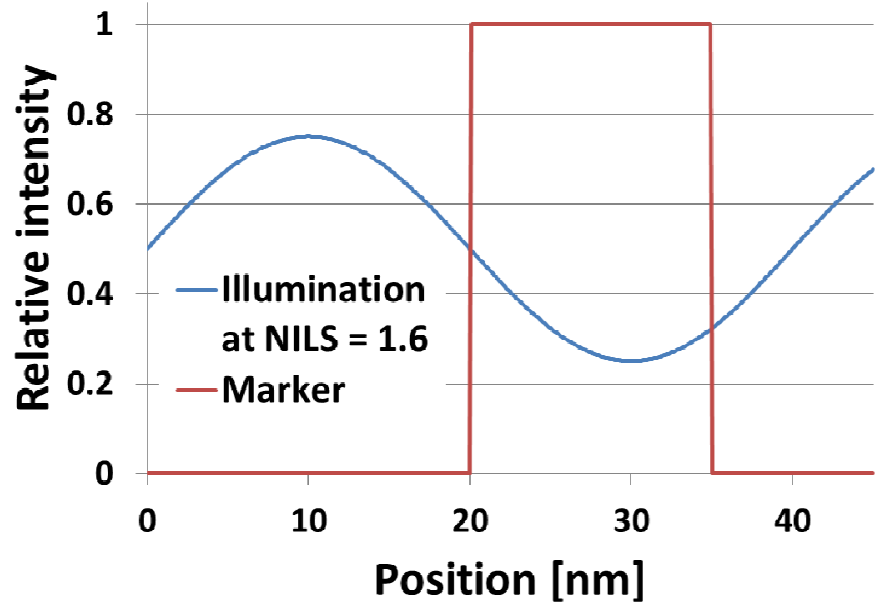

Figure 5. Example of one period of exposure light (blue line) with a NILS of 1.6 and the localized scintillating marker material (red line). The marker has the same pitch but a reduced width as compared to the expose beam modulation. 


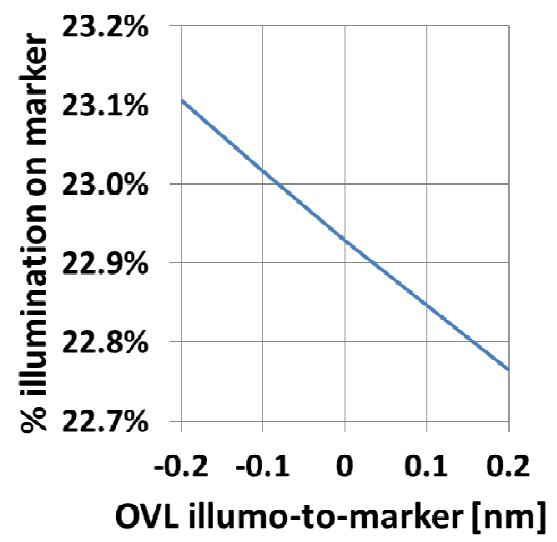

Figure 6. The fraction of the illumination light with a $40 \mathrm{~nm}$ pitch that hits a $15 \mathrm{~nm}$ marker as function of misalignment between illumination and marker for a NILS of 1.6.

\section{DISCUSSION}

To realize the proposed method for real-time overlay in practice, several hurdles have to be taken. Firstly, a IC manufacturer has to embrace the idea. The next step is to execute more advanced simulations of the obtainable signal strengths for a realistic marker design and relate those to the achievable OVL and focus accuracies. With these specifications, a decision can be made if it is worth to implement the method. Foreseen next engineering steps would comprise the identification of process-compatible flourophores as well as all modifications that are required to the scanner, the track and the additional processing. For this second phase, probably a consortium of equipment and IC manufactures is to be established.

\section{CONCLUSIONS}

A method for real-time overlay measurement is proposed. The heart of the invention lies in the introduction of a fluorescent marker that is patterned at product pitch on the wafer, and therefore is also sensitive to overlay shifts induced by e.g. lens heating. Before practical implementation of the method, quite some engineering effort is still required. The changes to the exposure process, stepper design and reticle lay-out for a practical realization of the proposed method were briefly discussed. Photon budget calculations predict a best-case overlay measurement accuracy of the method of better than $0.1 \mathrm{~nm}(3 \sigma)$.

\section{REFERENCES}

[1] W.H. Arnold, "Metrology in times of shrinking budgets", Proc. SPIE 8681 (2013) 868102

[2] C.-M. Ke, G.-T. Huang, J. Huang, J. and R. Lee, “Accuracy of diffraction-based and image-based overlay”, Proc. SPIE 7971 (2011) $79711 \mathrm{E}$

[3] N.M. Felix, A.H. Gabor, V.C. Menon, P.P. Longo, S.D. Halle, C.S. Koay, M.E. Colburn, "Overlay Improvement Roadmap: Strategies for Scanner Control and Product Disposition for $5 \mathrm{~nm}$ overlay”, Proc. SPIE 7971 (2011) $79711 \mathrm{D}$

[4] J. Mulkens, P. Hinnen, M. Kubis, A. Padiy, J. Benschop, "Holistic optimization architecture enabling sub-14-nm projection lithography”, J. Micro/Nanolith. MEMS MOEMS. 13, 011006 (2014)

[5] A.S. Lexmond, E.J. van Zwet and D.J. Maas, "System and method for overlay control", EP2602663

[6] T.A. Brunner and S.D. Smith, "Moiré technique for overlay metrology", Proc. SPIE 480 (1984) 164-170

[7] T.A. Brunner and R.R. Allen, "In situ measurement of an image during lithographic exposure", IEEE device letters 6 (1985) 329-331

[8] T.A. Brunner, S. Cheng and A.E. Norton, "A stepper image monitor for precise setup and characterization", Proc. SPIE 922 (1988) 366-375 
[9] M. Maenhoudt, J. Versluijs, H. Struyf, J. Van Olmen and M. Van Hove, "Double patterning scheme for sub-0.25 k1 single damascene structures at NA $=0.75, \lambda=193$ nm", Proc. SPIE 5754 (2005) 1508

[10] M.A. McCord, P. Petric, U. Ummethala, A. Carroll S. Kojima, L. Grella, S. Shriyan, C.T. Rettner, C.F. Bevis, "REBL: design progress toward $16 \mathrm{~nm}$ half-pitch maskless projection electron beam lithography", Proc. SPIE 8323 (2012) 832311; http://dx.doi.org/10.1117/12.919744

[11] H. Butler, "Adaptive feed-forward for a wafer stage in a lithographic tool", IEEE Transactions on Control Systems Technology Volume 21 (2013), 6170573, P.875-881

[12] Y. Blancquaert, C. Dezauzier, "Diffraction based overlay and image based overlay on production flow for advanced technology node", Proc. SPIE 8681 (2013) 868120

[13] K. Bhattacharyya, et. al., "New approaches for scatterometry-based metrology for critical distance and overlay measurement and process control”, J. Micro/Nanolith. MEMS MOEMS 10 (2011) 013013 